\title{
A 31 Year-old Man With Chest Pain, Rash, and EKG Changes
}

Neerav Sheth, MD, Rahul Anand, MD, and Matthew Stopper, MD

\section{Case Presentation}

A 31 year-old Caucasian male with no significant past medical history presents with increasing dizziness, chest pain, and shortness of breath of one day duration. On returning from a camping trip to Northern Pennsylvania three weeks prior to admission, he noted high fevers, "joint pains all over," and a pink blotchy rash all over his chest and back. He did not recall any insect, tick or animal bites. His primary physician, who clinically suspected Lyme disease, ordered Lyme serologies and offered a prescription for doxycycline which the patient declined. Two weeks later, the patient presented to the emergency department after the onset of chest pain that he described as sharp, radiating to both arms, occurring with exertion and relieved by rest. The chest pain was associated with worsening shortness of breath and dizziness.

The patient denied taking any medications, herbal or other supplements. He admitted to smoking half a pack of cigarettes per day, drinking 20 alcoholic beverages per week and occasionally smoking marijuana. He denied any intravenous drug use. He also denied any recent travel outside the United States or exposures to new chemicals or pets. The patient's mother had leukemia; his father had hypertension, diabetes, and hyperlipidemia; and his brother died from an anaphylactic reaction to shellfish.

On physical exam, the patient had a temperature of $99.6^{\circ}$ Fahrenheit, heart rate of 50 beats/minute, blood pressure of $155 / 77 \mathrm{~mm} \mathrm{Hg}$, respiratory rate of 14 breaths/minute with an oxygen saturation of $100 \%$ on room air. The exam also revealed a regularly irregular rhythm with no murmurs, rubs or gallops. His lungs were clear to auscultation bilaterally. He had no focal neurological deficits nor was he orthostatic. Large, well demarcated macular erythematous lesions were noted on his back and thorax. Initial labs showed a white blood cell count of 10,000 cells/mm 3 with a normal differential, normal serum chemistries, and a negative troponin. Peripheral smear was negative for babesiosis. The patient's admission chest radiograph showed clear lungs without evidence of infiltrate or effusion. His initial EKG (Figure 1) showed a sinus rhythm at an atrial rate of approximately 100 beats/minute and a highgrade atrioventricular (AV) block with a variable ventricular response of about 40 beats/minute. The patient was admitted to telemetry, and a transcutaneous pacer was placed. Empiric intravenouis ceftriaxone was begun to treat for a clinical diagnosis of Lyme carditis.

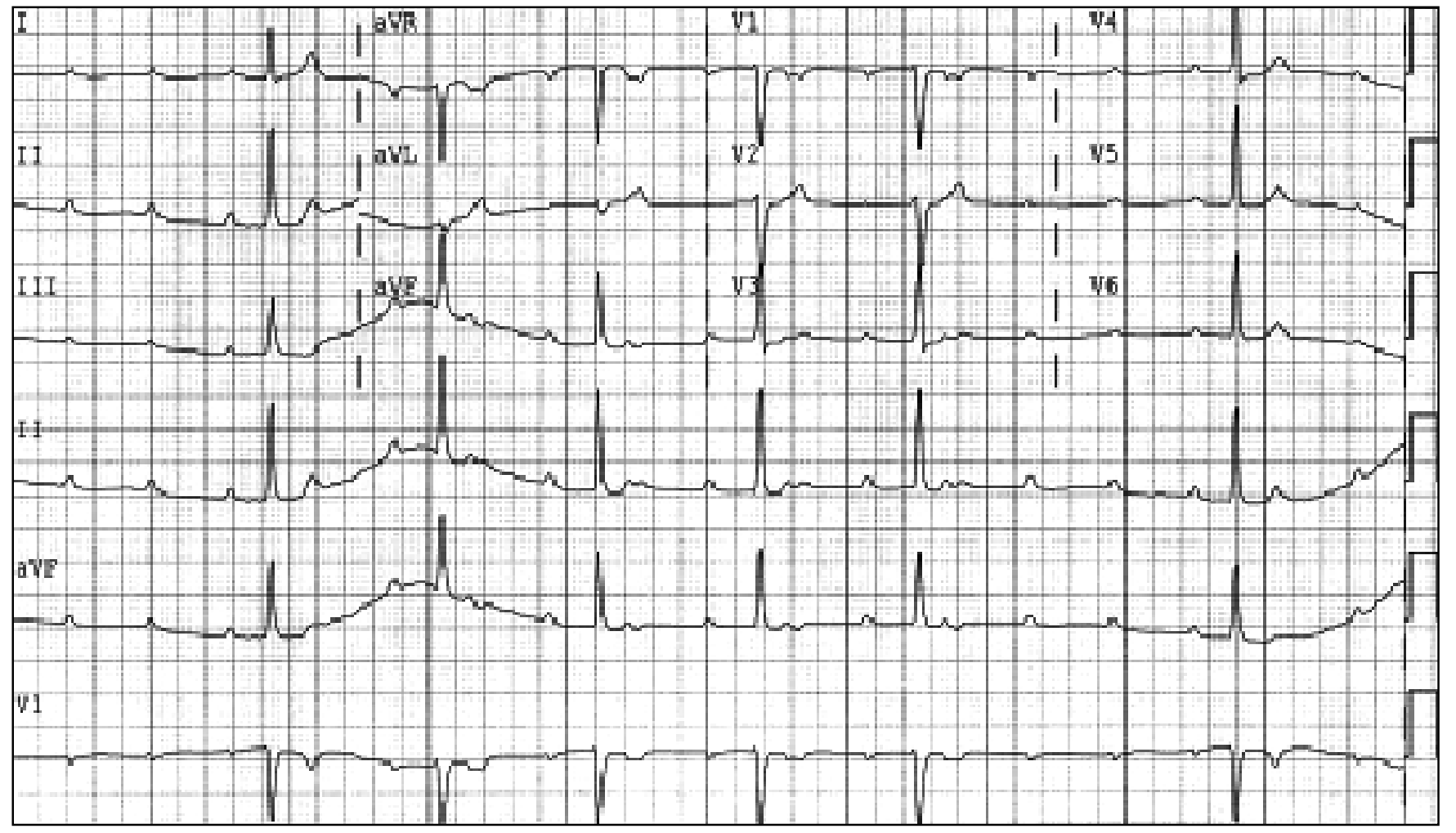

Figure 1. The patient's admission EKG showed high grade AV block with variable conduction. 


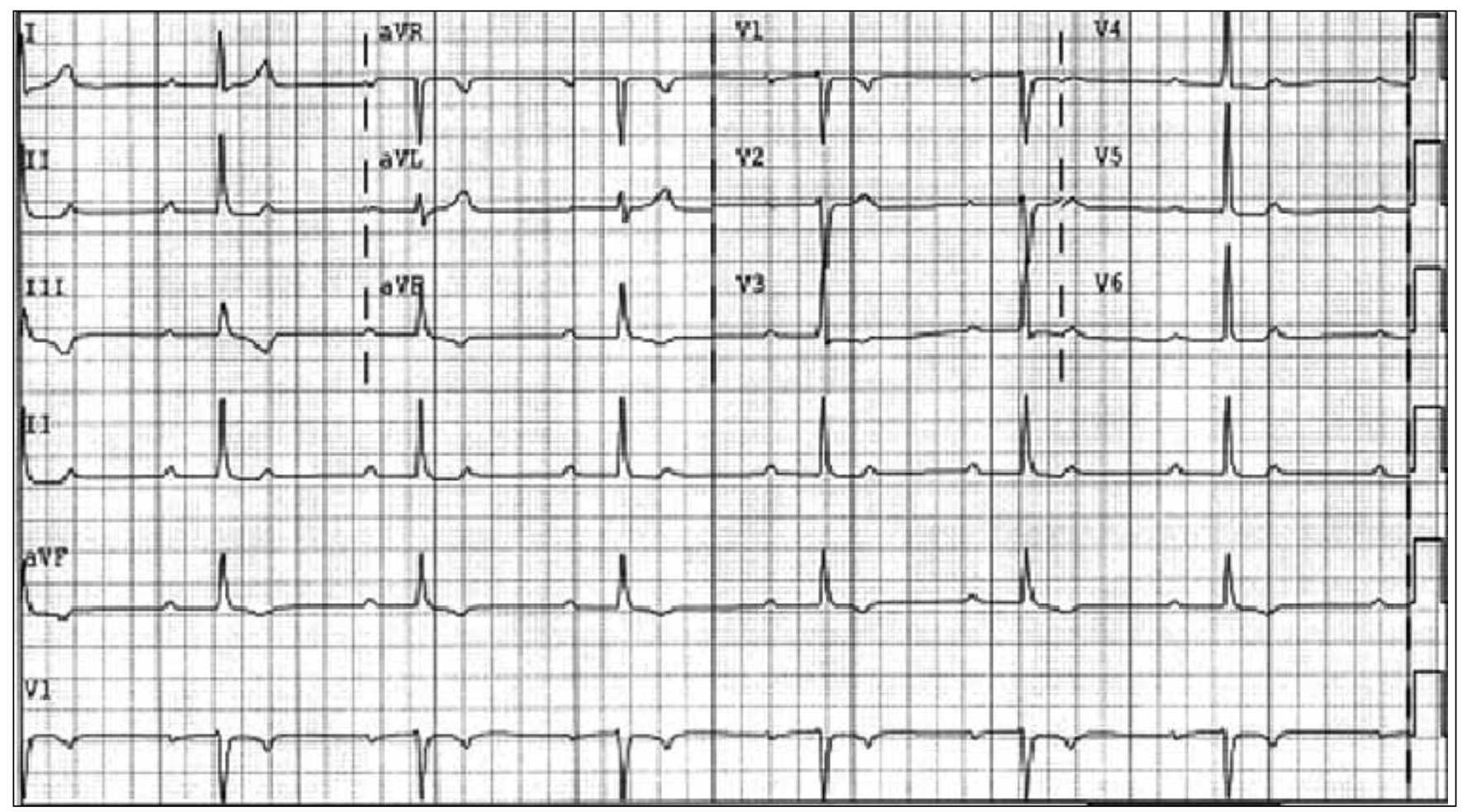

Figure 2. EKG on hospital day two showed second degree AV Block with 2:1 conduction.

Lyme IgM and IgG antibody titers were positive, but Lyme PCR was negative. A transthoracic echocardiogram revealed mild mitral regurgitation, normal left ventricular size and thickness with hyperdynamic function. His ejection fraction was approximately $85 \%$. By day two of hospitalization, his EKG had changed to sinus rhythm and second degree AV Block with 2:1 conduction (Figure 2). He was treated with intravenous ceftriaxone for 28 days with follow up in the cardiologic clinic two weeks after discharge. The repeat EKG at the follow up visit showed a return to normal sinus rhythm (Figure 3).

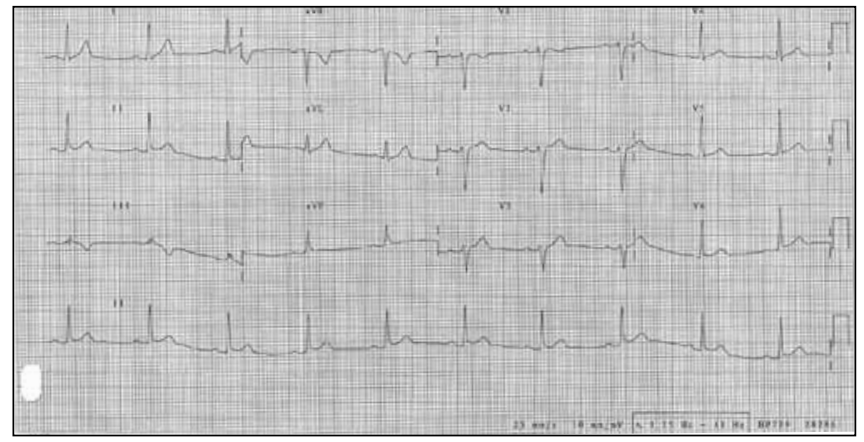

Figure 3. The patient's follow up EKG illustrates normal sinus rhythm.

\section{Discussion}

Lyme disease is a multi-system spirochetal infection caused by Borrelia burgdorferi affecting up to 20,000 Americans each year. A Diagnosis of Lyme disease requires a high index of suspicion and a good history. The majority of patients will report travel to an endemic region, but many will not recall a tick bite. ${ }^{1,2,3}$ Upon exposure, patients enter an asymptomatic incubation phase (lasting from three to 32 days). This phase is identified only by a localized skin reaction at the site of inoculation that is secondary to spirochetal infiltration of the dermis and epidermis. ${ }^{3}$ Following this, patient presentation varies depending on the phase of the disease. Table 1 depicts the symptoms associated with subsequent phases of Lyme disease if left untreated.

Our patient's symptomatology places him in the "Early Disseminated Phase," presenting with fluctuating AV block ranging from first degree heart block to high grade AV dissociation with variable conduction. Cardiovascular complications may occur in up to $8 \%$ of patients with untreated Lyme disease. ${ }^{10} \mathrm{AV}$ block is the most common cardiovascular manifestation of Lyme disease, attributed to spirochetal infiltration of the conduction system. ${ }^{4} \mathrm{AV}$ block secondary to Lyme disease is considered to be transient and is expected to resolve with treatment of the underlying disease. ${ }^{5}$

While AV block is the most common cardiovascular manifestation of Lyme disease, ${ }^{6}$ patients may also present with 
Table 1. Clinical Features of Lyme disease

\begin{tabular}{|l|l|l|l|}
\hline System & $\begin{array}{l}\text { Early Localized Phase } \\
\text { (months to years) }\end{array}$ & $\begin{array}{l}\text { Early Disseminated Phase } \\
\text { (days to weeks) }\end{array}$ & $\begin{array}{l}\text { Late Disseminated or Chronic } \\
\text { (weeks to months) }\end{array}$ \\
\hline Cardiac & (none) & $\begin{array}{l}\text { Atrioventricular block; myopericarditis; } \\
\text { pancarditis }\end{array}$ & (none) \\
\hline Constitutional & Influenza-like symptoms & Malaise; fatigue & Fatigue \\
\hline Lymphatic & Regional lymphadenopathy & $\begin{array}{l}\text { Regional or generalized lymphade- } \\
\text { nopathy }\end{array}$ & (none) \\
\hline Musculoskeletal & Myalgia & $\begin{array}{l}\text { Migratory pain in joints, bone, muscle; } \\
\text { brief arthritis attacks }\end{array}$ & $\begin{array}{l}\text { Prolonged oligoarticular arthritis } \\
\text { with frequent flares }\end{array}$ \\
\hline Neurologic & Headache & $\begin{array}{l}\text { Meningitis; Encephalitis, } \\
\text { Meningoencephalitis; Bell's palsy; } \\
\text { cranial neuritis; radiculoneuritis }\end{array}$ & $\begin{array}{l}\text { Encephalopathy; polyneuropathy; } \\
\text { leukoencephalitis; Dementia; Mood } \\
\text { Disorder }\end{array}$ \\
\hline \multicolumn{2}{|l|}{ Skin } & $\begin{array}{l}\text { Lecondary annular lesions } \\
\text { Erythema migrans }\end{array}$ & $\begin{array}{l}\text { Lymphocytoma; acrodermatitis } \\
\text { chronica atrophicans }\end{array}$ \\
\hline Adapted from Steere AC. Lyme disease. N Engl J Med 1989;321:589. & \\
\hline
\end{tabular}

myopericarditis, left ventricular dysfunction, cardiomegaly, pancarditis, aortitis, as well as ventricular tachyarrythmias. ${ }^{4,7,8}$ The exact reason for variability in presentation is unknown, but is theorized to be secondary to the location and degree of spirochetal infiltration as evidenced by cardiac biopsies of nonhuman primates with Lyme disease. ${ }^{9}$ Histopathologic stains of these biopsies show infiltration of the Borrelia spirochete from all regions of the heart, including aorta, atrium, and myocardium. ${ }^{9}$

Because of the potential for life-threatening complications, hospitalization and continuous monitoring are advisable for symptomatic patients (e.g., those with syncope, dyspnea, or chest pain). These interventions are also suggested for patients with second or third degree AV block, as well as for those with first degree heart block when the PR interval is prolonged by more than 30 milliseconds because the degree of block may fluctuate and worsen very rapidly in such patients. ${ }^{12}$

Patients with AV heart block and/or myopericarditis associated with early Lyme disease may be treated with either oral or parenteral antibiotic therapy for 14 days (range 14 to 21 days). For hospitalized patients, a parenteral antibiotic, such as ceftriaxone, is recommended as initial treatment, although there are no clinical trials to support this recommendation (Level of Evidence B-III). ${ }^{1}$ For patients with advanced heart block, a temporary pacemaker may be required; expert consultation with a cardiologist is recommended. The pacemaker may be discontinued when the advanced heart block has resolved. An oral antibiotic regimen should be used for completion of therapy and for outpatients, as is used for patients with erythema migrans without carditis. ${ }^{1}$

\section{Conclusion}

Patients with Lyme disease are often diagnosed and treated early in their disease course, and therefore, most will not progress to systemic complications. Untreated Lyme disease has a variable presentation, and a high index of suspicion along with a thorough history are required to diagnose patients. While patients with cardiovascular complications of Lyme disease, as in this case, will require a minimum of 28 days of antibiotics, they may also expect significant improvement in their symptoms.

\section{References}

1. Wormser, Gary et al. The Clinical Assessment, Treatment, and Prevention of Lyme Disease, Human Granulocytic Anaplasmosis, and Babesiosis" Clinical Infectious Diseases 2006; 43:1089-134)

2. Bratton RL, Whiteside JW, Hovan MJ, Engle RL, Edwards FD. Diagnosis and treatment of Lyme disease. Mayo Clin Proc. 2008 May;83(5):566-71. Review.

3. "Learn About Lyme Disease." Centers for Disease Control. 2008. www.cdc.gov/ncidod/dvbid/lyme

4. Lelovas P, Dontas I, Bassiakou E, Xanthos T.Cardiac implications of Lyme disease, diagnosis and therapeutic approach. Int J Cardiol. 2008 Sep 16;129(1):1521. Epub 2008 May 27.

5. Manzoor K, Aftab W, Choksi S, Khan IA.Lyme carditis: Sequential electrocardiographic changes in response to antibiotic therapy. Int J Cardiol. 2008 Aug 4. [Epub ahead of print]

6. van der Linde MR, Crijns HJ, de Koning J, Hoogkamp-Korstanje JA, de Graaf JJ, Piers DA, van der Galiën A, Lie KI. Range of atrioventricular conduction disturbances in Lyme borreliosis: a report of four cases and review of other published reports. Br Heart J. 1990 Mar;63(3):162-8. Review.

7. Cox J, Krajden M. Cardiovascular manifestations of Lyme disease. Am Heart J 1991 Nov;122(5):1449-55. Review.

8. Vijay, SC, Dervan, J.P., Elias, J., Kane, P.P., Dattwyler, R. Ventricular tachycardia associated with Lyme carditis. Am Heart J. 1991 May;121(5):1558-60.

\section{Acknowledgements:}

Stuart J. Gould, MD, Lan Quang, MD, and Samir Dalia, MD 